\title{
Violation of a simple factorized form of QCD amplitudes and Regge cuts
}

\author{
V.S. Fadin* \\ Author affiliation \\ Budker Institute of Nuclear Physics of SD RAS, 630090 Novosibirsk Russia \\ Novosibirsk State University, 630090 Novosibirsk, Russia \\ E-mail: fadineinp.nsk.su
}

\begin{abstract}
A simple factorized form of high energy QCD amplitudes with gluon quantum numbers in the cross channels and negative signature is known to be violated in the next-to-next-to-leading logarithmic approximation (NNLLA). The violation is explained by contributions of three-Reggeon cuts. The contributions of the cuts to the elastic amplitudes and the consequent need to change the scheme of derivation of the BFKL equation in the NNLLA is discussed.
\end{abstract}

XXV International Workshop on Deep-Inelastic Scattering and Related Subjects 3-7 April 2017

University of Birmingham, $U K$

\footnotetext{
* Speaker.
} 


\section{Introduction}

One of remarkable properties of QCD is the gluon Reggeization [1]. The Reggeization allows to express an infinite number of amplitudes through several Reggeon vertices and the Reggeized gluon trajectory. It underlies the BFKL (Balitsky-Fadin-Kuraev-Lipatov) approach [2, 3, 4, 5] and provides a general way for theoretical description of processes at high c.m.s. energy $\sqrt{s}$ and fixed (not growing with energy) momentum transfers.

The Reggeization means definite form not only of elastic amplitudes, but of inelastic amplitudes in the multi-Regge kinematics (MRK) as well. This kinematics is very important because it gives dominant contributions to cross sections, as well as to non-forward amplitude discontinuities in the unitarity relations. In this kinematics all particles have limited (not growing with $s$ ) transverse momenta and are combined into jets with limited invariant mass of each jet and large (growing with $s$ ) invariant masses of any pair of the jets. In the LLA each jet contains only one gluon; in the NLA one has to account production of $Q \bar{Q}$ and $G G$ jets.

Validity of the Reggeization is proved now in all orders of perturbation theory in the coupling constant $g$ both in the leading logarithmic approximation (LLA) [6], where in each order of the perturbation theory only terms with the highest powers of $\ln s$ are kept, and in the next-to-leading one (NLLA), where terms with one power less are also kept (see e.g. [7, 8] and references therein).

Extremely important that in these approximations elastic amplitudes with negative signature (symmetry with respect to the replacement $s \leftrightarrow u \simeq s$ ) and real parts of inelastic amplitudes in the MRK are determined by the Regge pole contributions and have a simple factorized form, which we will call pole Regge form. Due to this, the Reggeization provides a simple derivation of the BFKL equation in the LLA and the NLLA.

The pole Regge form is violated in the NNLLA. The first observation of the violation was done [9] at consideration of the high-energy limit of the two-loop amplitudes for for $g g, g q$ and $q q$ scattering. The discrepancy appears in non-logarithmic two-loop terms. If the pole Regge form would be correct in the NNLLA, they should satisfy a definite condition, because three amplitudes should be expressed in terms of two Reggeon-Particle-Particle vertices.

Detailed consideration of the terms responsible for breaking of the pole Regge form in the case of two-loop and three-loop quark and gluon amplitudes in QCD was performed in [10, 11, 12]. In particular, the non-logarithmic double-pole contribution at two-loops was recovered and all nonfactorizing single-logarithmic singular contributions at three loops were found using the techniques of infrared factorization.

I must say that, in general, breaking the pole Regge form is not a surprise. It is well known that Regge poles generate Regge cuts. Moreover, in amplitudes with positive signature the Regge cuts appear already in the LLA. Therefore it was natural to expect that the observed violation is explained by contributions of the Regge cuts. As it was shown in [13, 14], this is actually so.

Below we discuss contributions of the Regge cuts to parton (quark and gluon) elastic scattering amplitudes with the same (negative) signature as the Reggeized gluon and the influence of the Regge cuts on the derivation of the BFKL equation. Recall that leading terms in the negative signature are real, whereas in the positive signature they are imaginary due to cancellation of leading logarithmic terms. 


\section{Contributions of Regge cuts to amplitudes of elastic processes}

Due to the signature conservation the cuts with the negative signature have to be 3-Reggeon ones. Since our Reggeon is the Reggeized gluon, the cuts start with the diagrams with three $t$ channels gluons. We consider contribution of these diagrams to amplitudes of parton (quark and gluon) scattering. In contrast to the Reggeon, which contributes only to amplitudes with the adjoint representation of the colour group (colour octet in QCD) in the $t$-channel, the three-Reggeon cuts can contribute to various representations. Possible representations for quark-quark and quark-gluon scattering are only singlet (1) and octet $(\mathbf{8})$ whereas for the gluon-gluon scattering there are also $\mathbf{1 0}$, $\mathbf{1 0}^{*}$ and 27. Taking into account Bose statistic for gluons, symmetry of the representations $\mathbf{1}$ and $\mathbf{2 7}$, antisymmetry $\mathbf{1 0}$ and $\mathbf{1 0}^{*}$ and existence both symmetric $\mathbf{8}_{\mathrm{s}}$ and antisymmetric $\mathbf{8}_{\mathbf{a}}$ representations for them, gives that besides the Reggeon channel amplitudes with negative signature there are in the representations $\mathbf{1}$ for quark-quark-scattering and in the representation $\mathbf{1 0}$ and $\mathbf{1 0}^{*}$ for the gluongluon scattering.

Consideration of the colour structures of the diagrams gives that the contributions of the diagrams with three $t$-channels gluons to amplitudes $A^{(R)}$ for the representation $R$ of the colour group in the $t$-channel and negative signature have the form (we omit helicity conservation sign $\delta_{\lambda_{A A^{\prime}}} \delta_{\lambda_{B B^{\prime}}}$ )

$$
A_{i j}^{(8)}=\left\langle A^{\prime}\left|T^{a}\right| A\right\rangle\left\langle B^{\prime}\left|T^{a}\right| B\right\rangle\left[C_{i j} A^{(e i k)}+\frac{N_{c}^{2}}{8}\left(A_{i j}^{s}+A_{i j}^{u}\right)\right],
$$

where $i j$ are $q q, q g$ and $g g$ for quark-quark, quark-gluon and gluon-gluon scattering correspondingly, $A_{i j}^{s}$ and $A_{i j}^{u}$ are the the contributions of the ladder diagrams in the $s$ and $u$ channels respectively with omitted colour group factors, $A^{(e i k)}$ is the sum of such contributions for all the diagrams,

$$
A_{g g}^{\left(10+10^{*}\right)}=\varepsilon_{A}^{a} \varepsilon_{A}^{* a^{\prime}} \varepsilon_{B}^{b} \varepsilon_{B}^{* b^{\prime}}\left\langle a a^{\prime}\left|\hat{\mathscr{P}}_{10}+\hat{\mathscr{P}}_{10^{*}}\right| b b^{\prime}\right\rangle \frac{-3}{4} N_{c} A^{(e i k)},
$$

where $\varepsilon_{A}^{a}$ is the gluon $A$ colour polarization vector, $\hat{\mathscr{P}}_{R}$ is the projection operator on the representation $R$,

$$
\left\langle a a^{\prime}\left|\hat{\mathscr{P}}_{10}+\hat{\mathscr{P}}_{10^{*}}\right| b b^{\prime}\right\rangle=\frac{1}{2}\left(\delta^{b a} \delta^{b^{\prime} a^{\prime}}-\delta^{b a^{\prime}} \delta^{b^{\prime} a}\right)-\frac{1}{N_{c}} f^{b b^{\prime} c} f^{a a^{\prime} c},
$$

and

$$
A_{q q}^{(1)}=\chi_{A^{\prime} \alpha^{\prime}}^{*} \chi_{A}^{\alpha} \chi_{B}^{\beta} \chi_{B^{\prime} \beta^{\prime}}^{*} \delta_{\alpha}^{\alpha^{\prime}} \delta_{\beta}^{\beta^{\prime}} \frac{\left(N_{c}^{2}-4\right)\left(N_{c}^{2}-1\right)}{16 N_{c}^{3}} A^{(e i k)},
$$

where $\chi_{A}^{\alpha}$ is the quark $A$ colour spinor.

The eikonal amplitude $A^{e i k}$ can be written as $A^{e i k}=g^{2}(s / t)\left(-4 \pi^{2} / 3\right) g^{4} \vec{q}^{2} A_{\perp}^{(3)}$, where $A_{\perp}^{(3)}$ is given by the integral in the transverse momentum space:

$$
\begin{gathered}
A_{\perp}^{(3)}=\int \frac{d^{2+2 \varepsilon} l_{1} d^{2+2 \varepsilon} l_{2}}{(2 \pi)^{2(3+2 \varepsilon)} \vec{l}_{1}^{2} \vec{l}_{2}^{2}\left(\vec{q}-\vec{l}_{1}-\vec{l}_{2}\right)^{2}}=3 C_{\Gamma}^{2} \frac{4}{\varepsilon^{2}} \frac{\left(\vec{q}^{2}\right)^{2 \varepsilon}}{\vec{q}^{2}} \frac{\Gamma^{2}(1+2 \varepsilon) \Gamma(1-2 \varepsilon)}{\Gamma(1+\varepsilon) \Gamma^{2}(1-\varepsilon) \Gamma(1+3 \varepsilon)}, \\
C_{\Gamma}=\frac{\Gamma(1-\varepsilon) \Gamma^{2}(1+\varepsilon)}{(4 \pi)^{2+\varepsilon} \Gamma(1+2 \varepsilon)} .
\end{gathered}
$$

Note that whereas the amplitudes (2.2) and (2.4) contain only the Regge cut contributions, in the colour octet amplitude (2.1) there are both pole and cut contributions. The colour group coefficients 
$C_{i j}$ are

$$
C_{q q}=\frac{1}{4}\left(-1+\frac{3}{N_{c}^{2}}\right), C_{q g}=\frac{1}{4}, C_{g g}=\frac{3}{2} .
$$

They don't satisfy the pole Regge factorization, which requires

$$
2 C_{q g}^{R}=C_{q q}^{R}+C_{g g}^{R} .
$$

Separation of pole and cut contributions can be done by consideration of radiative corrections.

The Reggeon and three-Reggeon cut contributions have different dependence on $s$. In the case of the Reggeized gluon it come solely from the Regge factor with the trajectory

$$
\omega(t)=-g^{2} N_{c} \vec{q}^{2} \int \frac{d^{2+2 \varepsilon} l}{2(2 \pi)^{(3+2 \varepsilon)} \vec{l}^{2}(\vec{q}-\vec{l})^{2}}=-g^{2} N_{c} C_{\Gamma} \frac{2}{\varepsilon}\left(\vec{q}^{2}\right)^{\varepsilon} .
$$

In the case of the three-Reggeon cut, one has to take into account the Reggeization of each of the three gluons and the interaction between them. For the first logarithmic correction, the Reggeization gives $\ln s$ with the coefficient $3 C_{R}$, where

$$
C_{R}=-g^{2} N_{c} C_{\Gamma} \frac{4}{3 \varepsilon}\left(\vec{q}^{2}\right)^{\varepsilon} \frac{\Gamma(1-3 \varepsilon) \Gamma(1+2 \varepsilon) \Gamma(1+3 \varepsilon)}{\Gamma(1-\varepsilon) \Gamma(1-2 \varepsilon) \Gamma(1+\varepsilon) \Gamma(1+4 \varepsilon)} .
$$

Interaction between two Reggeons with transverse momenta $\vec{l}_{1}$ and $\vec{l}_{2}$ and colour indices $c_{1}$ and $c_{2}$ is given by the real part of the BFKL kernel

$$
\left[\mathscr{K}_{r}\left(\vec{q}_{1}, \vec{q}_{2} ; \vec{k}\right)\right]_{c_{1} c_{2}}^{c_{1}^{\prime} c_{2}^{\prime}}=T_{c_{1} c_{1}^{\prime}}^{a} T_{c_{2}^{\prime} c_{2}}^{a} \frac{g^{2}}{(2 \pi)^{D-1}}\left[\frac{\vec{q}_{1}^{2} \vec{q}_{2}^{\prime 2}+\vec{q}_{2}^{2} \vec{q}_{1}^{\prime 2}}{\vec{k}^{2}}-\vec{q}^{2}\right]
$$

where $\vec{k}$ is the momentum transferred from one Reggeon to another in the interaction, $\vec{q}_{1}^{\prime}$ and $\vec{q}_{2}^{\prime}\left(c_{1}^{\prime}\right.$ and $c_{2}^{\prime}$ ) are the Reggeon momenta (colour indices) after the interaction, $\vec{q}_{1}^{\prime}=\vec{q}_{1}-\vec{k}, \vec{q}_{2}^{\prime}=\vec{q}_{2}+\vec{k}$, and $\vec{q}=\vec{q}_{1}+\vec{q}_{2}=\vec{q}_{1}^{\prime}+\vec{q}_{2}^{\prime}$.

For the colour structures which we are interested in, account of interactions between all pairs of the Reggeons leads to the colour coefficients which differs from the Born ones by the factors $\kappa_{R} N_{c}$, where $\kappa_{1}=(3 / 2), \kappa_{8}=1, \quad \kappa_{10+10^{*}}=1 / 2$. Therefore, the first order correction due to Reggeon interaction in the case of the three-Reggeon cut for the representation $R$ is presented as $\kappa_{R}\left(-4 C_{R}-C_{3}\right) \ln s$, where the $C_{R}$ and $C_{3}$ come correspondingly from the first two terms and the last term in the square brackets in (2.11),

$$
C_{3}=g^{2} N_{c} C_{\Gamma} \frac{32}{9 \varepsilon}\left(\vec{q}^{2}\right)^{\varepsilon} \frac{\Gamma(1-3 \varepsilon) \Gamma(1-\varepsilon) \Gamma^{2}(1+3 \varepsilon)}{\Gamma^{2}(1-2 \varepsilon) \Gamma(1+2 \varepsilon) \Gamma(1+4 \varepsilon)} .
$$

Thus, the first order correction in the case of the three-Reggeon cut is $\left(\left(3-4 \kappa_{R}\right) C_{R}-\kappa_{R} C_{3}\right) \ln s$, where $C_{R}$ and $C_{3}$ are given by (2.10) and (2.12) respectively.

In the octet representation there is a mixture of the Regge pole and the cut. The first order correction due to the cut is $\left(-C_{R}-C_{3}\right) \ln s$, whereas due to the pole is $\omega(t) \ln s$, where $\omega(t)$ is given by (2.9).

The coefficients $C_{i j}$ can be presented as the sum

$$
C_{i j}=C_{i j}^{R}+C_{i j}^{C}
$$


with $C_{i j}^{R}$ satisfying (2). The choice

$$
C_{g g}^{R}=3, C_{g g}^{C}=-\frac{3}{2}, C_{g q}^{R}=\frac{7}{4}, C_{g q}^{C}=-\frac{3}{2}, C_{q q}^{R}=\frac{1}{2}, C_{q q}^{C}=\frac{3\left(1-N_{c}^{2}\right)}{4 N_{c}^{2}} .
$$

is consistent with the results obtained in [12]

Evidently, the three-Reggeon cut gives contributions to all $2 \rightarrow n+2$ amplitudes in the MRK. They must be also found for further development of the BFKL approach.

\section{Impact on scheme of derivation of the BFKL equation}

Original scheme of derivation of the BFKL approach looks as follows. In the BFKL approach, two-to-two scattering amplitudes with all possible quantum numbers in the $t$-channel are calculated using the $s$-channel unitarity and analyticity. In the LLA and in the NLLA, only amplitudes having the pole Regge form contribute to unitarity relations. It permits to present the $s$-channel discontinuities of the processes $A+B \rightarrow A^{\prime}+B^{\prime}$ as the convolutions $\Phi_{A^{\prime} A} \otimes G \otimes \Phi_{B^{\prime} B}$, where the impact factors $\Phi_{A^{\prime} A}$ and $\Phi_{B^{\prime} B}$ describe transitions $A \rightarrow A^{\prime}$ and $B \rightarrow B^{\prime}$ due to interactions with Reggeized gluons, $G$ is the Green's function for two interacting Reggeized gluons with an operator form $\hat{\mathscr{G}}=e^{Y \mathscr{K}}$, where $Y=\ln \left(s / s_{0}\right), s_{0}$ is an energy scale, $\hat{\mathscr{K}}$ is the kernel of the BFKL equation. The impact factors and the BFKL kernel are expressed in terms of the Reggeon vertices and trajectory. Energy dependence of scattering amplitudes is determined by the BFKL kernel, which is universal (process independent). The kernel $\hat{\mathscr{K}}=\hat{\omega}_{\infty}+\hat{\omega}_{2}+\hat{\mathscr{K}}_{r}$ is expressed through the Regge trajectories $\hat{\omega}_{1}$ and $\hat{\omega}_{2}$ of two gluons and the "real part" $\hat{\mathscr{K}}_{r}$ describing production of particles in their interaction: $\hat{\mathscr{K}}_{r}=\hat{\mathscr{K}}_{G}+\hat{\mathscr{K}}_{Q \bar{Q}}+\hat{\mathscr{K}}_{G G}$. In the LLA only $\hat{\mathscr{K}}_{G}$ must be kept, because only gluons can be produced; in the NNLLA production of quark-antiquark $(Q \bar{Q})$ and gluon $(G G)$ pairs is also possible.

If this scheme were applicable, for derivation of the BFKL equation in the NNLLA would be sufficient to calculate three-loop corrections to the trajectory, two-loop corrections to $\hat{\mathscr{K}}_{G}$, one-loop corrections to $\hat{\mathscr{K}}_{Q \bar{Q}}$ and $\hat{\mathscr{K}}_{G G}$ and to find in the Born approximation two new contributions to $\hat{\mathscr{K}}_{r}$, $\hat{\mathscr{K}}_{Q \bar{Q} G}$ and $\hat{\mathscr{K}}_{G G Q}$, .

However, this scheme is based on the pole Regge form of amplitudes which is violated in the NNLLA. In this approximation two large logarithms can be lost in the product of two amplitudes in the unitarity condition used for derivation of the BFKL equation. It can be done losing either both logarithms in one of the amplitudes, or one logarithm in each of the amplitudes. In the first case one of the amplitudes is taken in the NNLLA and the other in the LLA. Since the amplitudes in the LLA are real, only real parts of the NNLLA amplitudes are important in this case. But even for these parts the the pole Regge form becomes inapplicable because of the contributions of the three-Reggeon cuts which appear in this approximation. Moreover, it becomes necessary to take into account amplitudes with colour representations in cross channels different from the octet. Since such representations are different for quark-quark, quark-gluon and gluon-gluon scattering, it violates universality of consideration.

The scheme is violated also because in the NNLLA it becomes necessary to take into account imaginary parts of amplitudes.

Consideration of many-particle states in the unitarity condition is an even more complicated problem. 


\section{Summary}

The gluon Reggeization is one of remarkable properties of QCD. Due to this property, QCD amplitudes with the gluon quantum numbers in cross channels and negative signature in the leading and next-to-leading logarithmic approximations have a simple factorized form (pole Regge form) - This is true not only for elastic amplitudes, but also for real parts of multiparticle production amplitudes in the multi-Regge-kinematics. It makes the derivation of the BFKL equation using the $s$-channel unitarity rather simple.

In the next-to next-to-leading logarithmic approximation this way of deriving the BFKL equation is greatly complicated for a number of reasons. The first is the violation of the pole Regge form of amplitudes with octet representation of the colour group in cross channels and negative signature by the Regge cuts. The second is need to take into account in the unitarity relations amplitudes with negative signature and the representations of the colour group different from octet, which also are given by the Regge cuts. And the third is need to take into account in the unitarity relations amplitudes with positive signature. Unfortunately, it violates universality of the derivation of the BFKL equation for various processes.

\section{References}

[1] L.N. Lipatov, Yad. Fiz. 23, 642-656 (1976) [Sov. J. Nucl. Phys. 23, 338-345 (1976)].

[2] V.S. Fadin, E.A. Kuraev, and L.N. Lipatov, Phys. Lett. B 60, 50-52 (1975).

[3] E.A. Kuraev, L.N. Lipatov, and V.S. Fadin, Zh. Eksp. Teor. Fiz. 71, 840-855 (1976) [Sov. Phys. JETP 44, 443-450 (1976)].

[4] E.A. Kuraev, L.N. Lipatov, and V.S. Fadin, Zh. Eksp. Teor. Fiz. 72, 377-389 (1977) [Sov. Phys. JETP 45, 199-207 (1977)].

[5] I.I. Balitsky, and L.N. Lipatov, Yad. Fiz. 28, 1597-1611 (1978) [Sov. J. Nucl. Phys. 28, 822-829 (1978)].

[6] Ya.Ya. Balitskii, L.N. Lipatov, and V.S. Fadin, "Regge Processes In Nonabelian Gauge Theories" (in Russian), in Materials of IV Winter School of LNPI (Leningrad, 1979), pp. 109-149.

[7] B. L. Ioffe, V. S. Fadin and L. N. Lipatov, Cambridge University Press, 2010, ISBN: 9781107424753

[8] V. S. Fadin, M. G. Kozlov, and A. V. Reznichenko, Phys. Rev. D 92 no.8, 085044 (2015).

[9] V. Del Duca and E. W. N. Glover, JHEP 0110 (2001) 035 [hep-ph/0109028].

[10] V. Del Duca, G. Falcioni, L. Magnea and L. Vernazza, Phys. Lett. B 732, 233 (2014).

[11] V. Del Duca, G. Falcioni, L. Magnea and L. Vernazza, PoS RADCOR 2013, 046 (2013).

[12] V. Del Duca, G. Falcioni, L. Magnea and L. Vernazza, JHEP 1502, 029 (2015).

[13] V. S. Fadin, AIP Conf. Proc. 1819 (2017) no.1, 060003 [arXiv:1612.04481 [hep-ph]].

[14] S. Caron-Huot, E. Gardi and L. Vernazza, JHEP 1706 (2017) 016 [arXiv:1701.05241 [hep-ph]]. 